\title{
Statistical approximation of modified Schurer-type $q$-Bernstein Kantorovich operators
}

\section{Qiu Lin*}

\section{Correspondence:} linqiulq@126.com

School of Mathematics and Statistics, Northeastern University at Qinhuangdao, Qinhuangdao, 066004, China

\begin{abstract}
New modified Schurer-type q-Bernstein Kantorovich operators are introduced. The local theorem and statistical Korovkin-type approximation properties of these operators are investigated. Furthermore, the rate of approximation is examined in terms of the modulus of continuity and the elements of Lipschitz class functions. MSC: 41A10; 41A25; 41A36
\end{abstract}

Keywords: modified Schurer-type q-Bernstein Kantorovich operators; local approximation; modulus of continuity; statistical approximation

\section{Introduction}

In 1987, Lupaş [1] introduced a $q$-analogue of Bernstein operators, and in 1997 another $q$ generalization of the Bernstein polynomials was introduced by Phillips [2]. After that generalizations of the Bernstein polynomials based on the $q$-integers attracted a lot of interest and were studied widely by a number of authors. Some new generalizations of well-known positive linear operators based on $q$-integers were introduced and studied by several authors (e.g., see [3-6]). On the other hand, the study of the statistical convergence for sequences of positive operators was attempted by Gadjiev and Orhan [7]. Very recently, the statistical approximation properties have also been investigated for $q$-analogue polynomials. For instance, in [8] $q$-Bleimann, Butzer and Hahn operators; in [9] Kantorovich-type $q$-Bernstein operators; in [10] a $q$-analogue of MKZ operators; in [11] Kantorovich-type $q$-Szász-Mirakjan operators; in [12] Kantorovich-type $q$-Bernstein-Stancu operators were introduced and their statistical approximation properties were studied.

The paper is organized as follows. In Section 2, we introduce a new modification of Schurer-type $q$-Bernstein Kantorovich operators and evaluate the moments of these operators. In Section 3 we study local convergence properties in terms of the first and the second modulus of continuity. In Section 4, we obtain their statistical approximation properties with the help of the Korovkin-type theorem proved by Gadjiev and Orhan. Furthermore, in Section 5, we compute the degree of convergence of the approximation process in terms of the modulus of continuity and the Lipschitz class functions.

\section{Construction of the operators}

Some definitions and notations regarding the concept of $q$-calculus can be found in [5]. Let $\alpha, \beta, p \in \mathbb{N}^{0}$ (the set of all nonnegative integers) be such that $0 \leq \alpha \leq \beta$. We introduce a

\section{空 Springer}

(c)2014 Lin; licensee Springer. This is an Open Access article distributed under the terms of the Creative Commons Attribution License (http://creativecommons.org/licenses/by/2.0), which permits unrestricted use, distribution, and reproduction in any medium, provided the original work is properly cited. 
Lin Journal of Inequalities and Applications 2014, 2014:465

Page 2 of 11

http://www.journalofinequalitiesandapplications.com/content/2014/1/465

new modification of Schurer-type $q$-Bernstein Kantorovich operators $K_{n, q}^{(\alpha, \beta)}(f ; x): C[0,1+$ $p] \rightarrow C[0,1]$ as follows:

$$
K_{n, q}^{(\alpha, \beta)}(f ; x)=\sum_{k=0}^{n+p} \bar{p}_{n, k}(q ; x) \int_{0}^{1} f\left(\frac{t}{[n+1+\beta]_{q}}+\frac{q[k+\alpha]_{q}}{[n+1+\beta]_{q}}\right) d_{q} t
$$

where $x \in[0,1]$ and $\bar{p}_{n, k}(q ; x)=\left[\begin{array}{c}n+p \\ k\end{array}\right] q^{x^{k}} \prod_{s=0}^{n+p-k-1}\left(1-q^{s} x\right)$. It is clear that $K_{n, q}^{(\alpha, \beta)}(f ; x)$ is a linear and positive operator. When $\alpha=\beta=0$, it reduces to the Schurer-type $q$-Bernstein Kantorovich operators (see [13])

$$
K_{n}^{p}(f ; q ; x)=\sum_{k=0}^{n+p}\left[\begin{array}{c}
n+p \\
k
\end{array}\right]_{q} x^{k} \prod_{s=0}^{n+p-k-1}\left(1-q^{s} x\right) \int_{0}^{1} f\left(\frac{t}{[n+1]_{q}}+\frac{q[k]_{q}}{[n+1]_{q}}\right) d_{q} t .
$$

In order to investigate the approximation properties of $K_{n, q}^{(\alpha, \beta)}$, we need the following lemmas.

Lemma 2.1 ([14]) For the generalized q-Schurer-Stancu operators

$$
\left(S_{n, p, q}^{(\alpha, \beta)} f\right)(x)=\sum_{k=0}^{n+p}\left[\begin{array}{c}
n+p \\
k
\end{array}\right]_{q} x^{k} \prod_{s=0}^{n+p-k-1}\left(1-q^{s} x\right) f\left(\frac{[k+\alpha]_{q}}{[n+\beta]_{q}}\right), \quad x \in[0,1],
$$

the following properties hold:

$$
\begin{aligned}
& \left(S_{n, p, q}^{(\alpha, \beta)} 1\right)(x)=1 \\
& \left(S_{n, p, q}^{(\alpha, \beta)} t\right)(x)=\frac{q^{\alpha}[n+p]_{q}}{[n+\beta]_{q}} x+\frac{[\alpha]_{q}}{[n+\beta]_{q}} \\
& \left(S_{n, p, q}^{(\alpha, \beta)} t^{2}\right)(x)=\frac{[n+p]_{q}[n+p-1]_{q}}{[n+\beta]_{q}^{2}} q^{2 \alpha+1} x^{2}+\frac{[n+p]_{q} q^{\alpha}}{[n+\beta]_{q}^{2}}\left(2[\alpha]_{q}+q^{\alpha}\right) x+\frac{[\alpha]_{q}^{2}}{[n+\beta]_{q}^{2}}
\end{aligned}
$$

Lemma 2.2 For $K_{n, q}^{(\alpha, \beta)}\left(t^{i} ; x\right), i=0,1,2$, we have

$$
\begin{aligned}
K_{n, q}^{(\alpha, \beta)}(1 ; x)= & 1 \\
K_{n, q}^{(\alpha, \beta)}(t ; x)= & \frac{[n+p]_{q}}{[n+1+\beta]_{q}} q^{\alpha+1} x+\frac{1}{[n+1+\beta]_{q}}\left(\frac{1}{[2]_{q}}+q[\alpha]_{q}\right), \\
K_{n, q}^{(\alpha, \beta)}\left(t^{2} ; x\right)= & \frac{[n+p]_{q}[n+p-1]_{q}}{[n+1+\beta]_{q}^{2}} q^{2 \alpha+3} x^{2} \\
& +\frac{[n+p]_{q}}{[n+1+\beta]_{q}^{2}}\left(\frac{2}{[2]_{q}} q^{\alpha+1}+q^{2+\alpha}\left(2[\alpha]_{q}+q^{\alpha}\right)\right) x \\
& +\frac{1}{[n+1+\beta]_{q}^{2}}\left(\frac{1}{[3]_{q}}+\frac{2 q[\alpha]_{q}}{[2]_{q}}+q^{2}[\alpha]_{q}^{2}\right) .
\end{aligned}
$$

Proof It is obvious that

$$
\int_{0}^{1} 1 d_{q} t=1, \quad \int_{0}^{1} t d_{q} t=\frac{1}{[2]_{q}}, \quad \int_{0}^{1} t^{2} d_{q} t=\frac{1}{[3]_{q}}, \quad 0<q<1 .
$$


For $i=0$, since $\sum_{k=0}^{n+p} \bar{p}_{n, k}(q ; x)=1$, so (2.5) holds.

For $i=1$, we get

$$
\begin{aligned}
K_{n, q}^{(\alpha, \beta)}(t ; x) & =\sum_{k=0}^{n+p} \bar{p}_{n, k}(q ; x) \int_{0}^{1}\left(\frac{t}{[n+1+\beta]_{q}}+\frac{q[k+\alpha]_{q}}{[n+1+\beta]_{q}}\right) d_{q} t \\
& =\sum_{k=0}^{n+p} \bar{p}_{n, k}(q ; x) \int_{0}^{1} \frac{t}{[n+1+\beta]_{q}} d_{q} t+\sum_{k=0}^{n+p} \bar{p}_{n, k}(q ; x) \int_{0}^{1} \frac{q[k+\alpha]_{q}}{[n+1+\beta]_{q}} d_{q} t \\
& =\frac{1}{[2]_{q}[n+1+\beta]_{q}}+\sum_{k=0}^{n+p} \bar{p}_{n, k}(q ; x) \frac{q[k+\alpha]_{q}}{[n+1+\beta]_{q}} .
\end{aligned}
$$

Using (2.3), we have

$$
\begin{aligned}
\sum_{k=0}^{n+p} \bar{p}_{n, k}(q ; x) \frac{q[k+\alpha]_{q}}{[n+1+\beta]_{q}} & =\frac{q[n+\beta]_{q}}{[n+1+\beta]_{q}} \sum_{k=0}^{n+p} \bar{p}_{n, k}(q ; x) \frac{[k+\alpha]_{q}}{[n+\beta]_{q}} \\
& =\frac{q[n+\beta]_{q}}{[n+1+\beta]_{q}}\left(S_{n, p, q}^{(\alpha, \beta)} t\right)(x) .
\end{aligned}
$$

So

$$
K_{n, q}^{(\alpha, \beta)}(t ; x)=\frac{[n+p]_{q}}{[n+1+\beta]_{q}} q^{\alpha+1} x+\frac{1}{[n+1+\beta]_{q}}\left(\frac{1}{[2]_{q}}+q[\alpha]_{q}\right) .
$$

For $i=2$,

$$
\begin{aligned}
& \int_{0}^{1}\left(\frac{t}{[n+1+\beta]_{q}}+\frac{q[k+\alpha]_{q}}{[n+1+\beta]_{q}}\right)^{2} d_{q} t \\
& \quad=\frac{1}{[n+1+\beta]_{q}^{2}}\left(\int_{0}^{1} t^{2} d_{q} t+2 q[k+\alpha]_{q} \int_{0}^{1} t d_{q} t+q^{2}[k+\alpha]_{q}^{2} \int_{0}^{1} 1 d_{q} t\right) \\
& \quad=\frac{1}{[n+1+\beta]_{q}^{2}}\left(\frac{1}{[3]_{q}}+\frac{2 q[k+\alpha]_{q}}{[2]_{q}}+q^{2}[k+\alpha]_{q}^{2}\right),
\end{aligned}
$$

we obtain

$$
\begin{aligned}
K_{n, q}^{(\alpha, \beta)}\left(t^{2} ; x\right) & =\frac{1}{[n+1+\beta]_{q}^{2}} \sum_{k=0}^{n+p} \bar{p}_{n, k}(q ; x)\left(\frac{1}{[3]_{q}}+\frac{2 q[k+\alpha]_{q}}{[2]_{q}}+q^{2}[k+\alpha]_{q}^{2}\right) \\
= & \frac{1}{[3]_{q}[n+1+\beta]_{q}^{2}}+\frac{[n+\beta]_{q}}{[n+1+\beta]_{q}^{2}} \frac{2 q}{[2]_{q}} \sum_{k=0}^{n+p} \bar{p}_{n, k}(q ; x) \frac{[k+\alpha]_{q}}{[n+\beta]_{q}} \\
& +\frac{q^{2}[n+\beta]_{q}^{2}}{[n+1+\beta]_{q}^{2}} \sum_{k=0}^{n+p} \bar{p}_{n, k}(q ; x) \frac{[k+\alpha]_{q}^{2}}{[n+\beta]_{q}^{2}} \\
= & \frac{1}{[3]_{q}[n+1+\beta]_{q}^{2}}+\frac{[n+\beta]_{q}}{[n+1+\beta]_{q}^{2}} \frac{2 q}{[2]_{q}}\left(S_{n, p, q}^{(\alpha, \beta)} t\right)(x)+\frac{q^{2}[n+\beta]_{q}^{2}}{[n+1+\beta]_{q}^{2}}\left(S_{n, p, q}^{(\alpha, \beta)} t^{2}\right)(x) .
\end{aligned}
$$

Using (2.3) and (2.4), by a simple calculation we can get the stated result (2.7). 
Lemma 2.3 From Lemma 2.2, we have

$$
\begin{aligned}
\mu_{n, q}^{p}(x) & :=K_{n, q}^{(\alpha, \beta)}(t-x ; x) \\
& =\left(\frac{q^{\alpha+1}[n+p]_{q}}{[n+1+\beta]_{q}}-1\right) x+\frac{1}{[n+1+\beta]_{q}}\left(\frac{1}{[2]_{q}}+q[\alpha]_{q}\right)
\end{aligned}
$$

and

$$
\begin{aligned}
\delta_{n, q}^{p}(x):= & K_{n, q}^{(\alpha, \beta)}\left((t-x)^{2} ; x\right) \\
= & \left(\frac{[n+p]_{q}[n+p-1]_{q} q^{2 \alpha+3}}{[n+1+\beta]_{q}^{2}}-\frac{2 q^{\alpha+1}[n+p]_{q}}{[n+1+\beta]_{q}}+1\right) x^{2} \\
& +\left(\frac{[n+p]_{q}}{[n+1+\beta]_{q}^{2}}\left(\frac{2}{[2]_{q}} q^{\alpha+1}+q^{2+\alpha}\left(2[\alpha]_{q}+q^{\alpha}\right)\right)\right. \\
& \left.-\frac{2}{[n+1+\beta]_{q}}\left(\frac{1}{[2]_{q}}+q[\alpha]_{q}\right)\right) x \\
& +\frac{1}{[n+1+\beta]_{q}^{2}}\left(\frac{1}{[3]_{q}}+\frac{2 q[\alpha]_{q}}{[2]_{q}}+q^{2}[\alpha]_{q}^{2}\right) .
\end{aligned}
$$

\section{Local approximation}

Now, we consider a sequence $q=q_{n}$ satisfying the following two expressions:

$$
\lim _{n \rightarrow \infty} q_{n}=1 \text { and } \quad \lim _{n \rightarrow \infty} \frac{1}{[n]_{q_{n}}}=0
$$

By the Korovkin theorem, we can state the following theorem.

Theorem 3.1 Let $K_{n, q_{n}}^{(\alpha, \beta)}(f ; x)$ be a sequence satisfying (3.1) for $0<q_{n}<1$. Then, for any function $f \in C[0, p+1]$, the following equality

$$
\lim _{n \rightarrow \infty}\left\|K_{n, q_{n}}^{(\alpha, \beta)}(f ; \cdot)-f\right\|_{C[0,1]}=0
$$

is satisfied.

Proof We know that $K_{n, q}^{(\alpha, \beta)}(f ; x)$ is linear positive. By Lemma 2.2, if we choose the sequence $q=q_{n}$ satisfying conditions (3.1), and using the equality

$$
[n+\alpha]_{q_{n}}=[n]_{q_{n}}+q_{n}^{n}[\alpha]_{q_{n}}, \quad[n+1+\beta]_{q_{n}}=[n]_{q_{n}}+q_{n}^{n}[\beta+1]_{q_{n}},
$$

we have

$$
K_{n, q_{n}}^{(\alpha, \beta)}(q ; x) \rightrightarrows 1, \quad K_{n, q_{n}}^{(\alpha, \beta)}(t ; x) \rightrightarrows x, \quad K_{n, q_{n}}^{(\alpha, \beta)}\left(t^{2} ; x\right) \rightrightarrows x^{2}
$$

as $n \rightarrow \infty$. Because of the linearity and positivity of $K_{n, q_{n}}^{(\alpha, \beta)}(f ; x)$, the proof is complete by the classical Korovkin theorem.

Consider the following $K$-functional:

$$
K_{2}\left(f, \delta^{2}\right):=\inf \left\{\|f-g\|+\delta^{2}\left\|g^{\prime \prime}\right\|: g \in C^{2}[0, p+1]\right\}, \quad \delta \geq 0,
$$


where

$$
C^{2}[0, p+1]:=\left\{g: g, g^{\prime}, g^{\prime \prime} \in C[0, p+1]\right\}
$$

Then, in view of a known result [15], there exists an absolute constant $C>0$ such that

$$
K_{2}\left(f, \delta^{2}\right) \leq C \omega_{2}(f, \delta)
$$

where

$$
\omega_{2}(f, \delta):=\sup _{0<h \leq \delta} \sup _{x \pm h \in[0, p+1]}|f(x-h)-2 f(x)+f(x+h)|
$$

is the second modulus of smoothness of $f \in C[0, p+1]$.

Let $f \in C[0, p+1]$, for any $\delta>0$, the usual modulus of continuity for $f$ is defined as $\omega(f, \delta)=\sup _{0<h \leq \delta} \sup _{x, x+h \in[0, p+1]}|f(x+h)-f(x)|$.

We next present the following local theorem of the operators $K_{n, q}^{(\alpha, \beta)}(f ; x)$ in terms of the first and the second modulus of continuity of the function $f \in C[0, p+1]$.

Theorem 3.2 Let $f \in C[0, p+1]$, there exists an absolute constant $C>0$ such that

$$
\left|K_{n, q}^{(\alpha, \beta)}(f ; x)-f(x)\right| \leq C \omega_{2}\left(f, \sqrt{a_{n, q}^{p}(x)}\right)+\omega\left(f, b_{n, q}^{p}(x)\right),
$$

where

$$
\begin{aligned}
& a_{n, q}^{p}(x) \\
& =\left(\frac{q^{2 \alpha+2}[n+p]_{q}^{2}}{[n+1+\beta]_{q}^{2}}+\frac{[n+p]_{q}[n+p-1]_{q}}{[n+1+\beta]_{q}^{2}} q^{2 \alpha+3}-\frac{4 q^{\alpha+1}[n+p]_{q}}{[n+1+\beta]_{q}}+2\right) x^{2} \\
& \quad+\left(\frac{[n+p]_{q}}{[n+1+\beta]_{q}^{2}}\left(\frac{4 q^{\alpha+1}}{[2]_{q}}+4 q^{\alpha+2}[\alpha]_{q}+q^{\alpha+2}\right)-\frac{4}{[n+1+\beta]_{q}}\left(\frac{1}{[2]_{q}}+q[\alpha]_{q}\right)\right) x \\
& \quad+\frac{1}{[n+1+\beta]_{q}^{2}}\left(2 q^{2}[\alpha]_{q}^{2}+\frac{4 q[\alpha]_{q}}{[2]_{q}}+\frac{1}{[2]_{q}^{2}}+\frac{1}{[3]_{q}}\right)
\end{aligned}
$$

and

$$
b_{n, q}^{p}(x)=\left|\left(\frac{q^{\alpha+1}[n+p]_{q}}{[n+1+\beta]_{q}}-1\right) x+\frac{1}{[n+1+\beta]_{q}}\left(\frac{1}{[2]_{q}}+q[\alpha]_{q}\right)\right| .
$$

Proof Let

$$
\bar{K}_{n, q}^{(\alpha, \beta)}(f ; x):=K_{n, q}^{(\alpha, \beta)}(f ; x)-f\left(\xi_{n, q}^{p}(x)\right)+f(x),
$$

where $f \in C[0, p+1]$ and

$$
\xi_{n, q}^{p}(x)=\frac{[n+p]_{q}}{[n+1+\beta]_{q}} q^{\alpha+1} x+\frac{1}{[n+1+\beta]_{q}}\left(\frac{1}{[2]_{q}}+q[\alpha]_{q}\right) .
$$


Using the Taylor formula

$$
g(t)=g(x)+g^{\prime}(x)(t-x)+\int_{x}^{t}(t-s) g^{\prime \prime}(s) d s
$$

for $g \in C^{2}[0, p+1]$, we have

$$
\bar{K}_{n, q}^{(\alpha, \beta)}(g ; x)=g(x)+K_{n, q}^{(\alpha, \beta)}\left(\int_{x}^{t}(t-s) g^{\prime \prime}(s) d s ; x\right)-\int_{x}^{\xi_{n, q}^{p}(x)}\left(\xi_{n, q}^{p}(x)-s\right) g^{\prime \prime}(s) d s .
$$

Hence

$$
\begin{aligned}
& \left|\bar{K}_{n, q}^{(\alpha, \beta)}(g ; x)-g(x)\right| \\
& \quad \leq K_{n, q}^{(\alpha, \beta)}\left(\left|\int_{x}^{t}\right| t-s|\cdot| g^{\prime \prime}(s)|d s| ; x\right)+\left|\int_{x}^{\xi_{n, q}^{p}(x)}\right| \xi_{n, q}^{p}(x)-s|\cdot| g^{\prime \prime}(s)|d s| \\
& \quad \leq\left\|g^{\prime \prime}\right\| K_{n, q}^{(\alpha, \beta)}\left((t-x)^{2} ; x\right)+\left\|g^{\prime \prime}\right\|\left(\xi_{n, q}^{p}(x)-x\right)^{2} .
\end{aligned}
$$

Observe that

$$
K_{n, q}^{(\alpha, \beta)}\left((t-x)^{2} ; x\right)+\left(\xi_{n, q}^{p}(x)-x\right)^{2}=a_{n, q}^{p}(x),
$$

we obtain

$$
\left|\bar{K}_{n, q}^{(\alpha, \beta)}(g ; x)-g(x)\right| \leq a_{n, q}^{p}(x)\left\|g^{\prime \prime}\right\|
$$

Using (3.4) and the uniform boundedness of $K_{n, q}^{(\alpha, \beta)}$, we get

$$
\begin{aligned}
\left|K_{n, q}^{(\alpha, \beta)}(f ; x)-f(x)\right| \leq & \left|\bar{K}_{n, q}^{(\alpha, \beta)}(f-g ; x)\right|+\left|\bar{K}_{n, q}^{(\alpha, \beta)}(g ; x)-g(x)\right| \\
& +|f(x)-g(x)|+\left|f\left(\xi_{n, q}^{p}(x)\right)-f(x)\right| \\
\leq & 4\|f-g\|+a_{n, q}^{p}(x)\left\|g^{\prime \prime}\right\|+\omega\left(f, b_{n, q}^{p}(x)\right) .
\end{aligned}
$$

Taking the infimum on the right-hand side over all $g \in C^{2}[0, p+1]$, we obtain

$$
\left|K_{n, q}^{(\alpha, \beta)}(f ; x)-f(x)\right| \leq C K_{2}\left(f, \sqrt{a_{n, q}^{p}(x)}\right)+\omega\left(f, b_{n, q}^{p}(x)\right),
$$

which together with (3.3) gives the proof of the theorem.

\section{Korovkin-type statistical approximation properties}

Further on, let us recall the concept of statistical convergence which was introduced by Fast [16].

Let the set $K \in N$ and $K_{n}=\{k \leq n: k \in K\}$, the natural density of $K$ is defined by $\delta(K):=$ $\lim _{n \rightarrow \infty} \frac{1}{n}\left|K_{n}\right|$ if the limit exists (see [17]), where $\left|K_{n}\right|$ denotes the cardinality of the set $K_{n}$.

A sequence $x=x_{k}$ is called statistically convergent to a number $L$ if for every $\varepsilon>0$, $\delta\left\{k \in N:\left|x_{k}-L\right| \geq \varepsilon\right\}=0$. This convergence is denoted as $s t-\lim _{k} x_{k}=L$. It is known that any convergent sequence is statistically convergent, but not conversely. Details can be found in [18]. 
In approximation theory by linear positive operators, the concept of statistical convergence was used by Gadjiev and Orhan [7]. They proved the following Bohman-Korovkintype approximation theorem for statistical convergence.

Theorem 4.1 ([7]) If the sequence of linear positive operators $A_{n}: C[a, b] \rightarrow C[a, b]$ satisfies the conditions

$$
\begin{gathered}
s t-\lim _{n}\left\|A_{n}\left(e_{i} ; \cdot\right)-e_{i}\right\|_{C[a, b]}=0 \\
\text { for } e_{i}(t)=t^{i}, i=0,1,2, \text { then, for any } f \in C[a, b], \\
s t-\lim _{n}\left\|A_{n}(f ; \cdot)-f\right\|_{C[a, b]}=0 .
\end{gathered}
$$

In this section, we establish the following Korovkin-type statistical approximation theorems.

Theorem 4.2 Let $q=q_{n}, 0<q_{n}<1$, be a sequence satisfying the following conditions:

$$
s t-\lim _{n} q_{n}=1, \quad s t-\lim _{n} q_{n}^{n}=a \quad(a<1) \quad \text { and } \quad s t-\lim _{n} \frac{1}{[n]_{q_{n}}}=0,
$$

then, for $f \in C[0, p+1]$, we have

$$
s t-\lim _{n}\left\|K_{n, q_{n}}^{(\alpha, \beta)}(f ; \cdot)-f\right\|_{C[0,1]}=0 .
$$

Proof From Theorem 4.1, it is enough to prove that $s t-\lim _{n}\left\|K_{n, q_{n}}^{(\alpha, \beta)}\left(e_{i} ; \cdot\right)-e_{i}\right\|_{C[0,1]}=0$ for $e_{i}=t^{i}, i=0,1,2$.

By (2.5), we can easily get

$$
s t-\lim _{n}\left\|K_{n, q_{n}}^{(\alpha, \beta)}\left(e_{0} ; \cdot\right)-e_{0}\right\|_{C[0,1]}=0 .
$$

From equality (2.8) and (3.2) we have

$$
\begin{aligned}
& \left\|K_{n, q_{n}}^{(\alpha, \beta)}\left(e_{1} ; \cdot\right)-e_{1}\right\|_{C[0,1]} \\
& \quad \leq\left|\frac{q_{n}^{\alpha+1}[n+p]_{q_{n}}}{[n+1+\beta]_{q_{n}}}-1\right|+\frac{1}{[n+1+\beta]_{q_{n}}}\left(\frac{1}{[2]_{q_{n}}}+q_{n}[\alpha]_{q_{n}}\right) \\
& \quad \leq\left|\frac{q_{n}^{\alpha+1}[n+p]_{q_{n}}}{[n+1+\beta]_{q_{n}}}-1\right|+\frac{1+\alpha}{[n]_{q_{n}}} .
\end{aligned}
$$

Now, for given $\varepsilon>0$, let us define the following sets:

$$
\begin{aligned}
& U=\left\{k:\left\|K_{n, q_{k}}^{(\alpha, \beta)}\left(e_{1} ; \cdot\right)-e_{1}\right\|_{C[0,1]} \geq \varepsilon\right\}, \\
& U_{1}=\left\{k: \frac{q_{k}^{\alpha+1}[n+p]_{q_{k}}}{[n+1+\beta]_{q_{k}}}-1 \geq \frac{\varepsilon}{2}\right\}, \\
& U_{2}=\left\{k: \frac{1+\alpha}{[n]_{q_{k}}} \geq \frac{\varepsilon}{2}\right\} .
\end{aligned}
$$


From (4.3), one can see that $U \subseteq U_{1} \cup U_{2}$, so we have

$$
\begin{aligned}
\delta\{k & \left.\leq n:\left\|K_{n, q_{k}}^{(\alpha, \beta)}\left(e_{1} ; \cdot\right)-e_{1}\right\|_{C[0,1]} \geq \varepsilon\right\} \\
& \leq \delta\left\{k \leq n: \frac{q_{k}^{\alpha+1}[n+p]_{q_{k}}}{[n+1+\beta]_{q_{k}}}-1 \geq \frac{\varepsilon}{2}\right\}+\delta\left\{k \leq n: \frac{1+\alpha}{[n]_{q_{k}}} \geq \frac{\varepsilon}{2}\right\} .
\end{aligned}
$$

By (4.1) it is clear that

$$
s t-\lim _{n}\left(\frac{q_{n}^{\alpha+1}[n+p]_{q_{n}}}{[n+1+\beta]_{q_{n}}}-1\right)=0
$$

and

$$
s t-\lim _{n} \frac{1+\alpha}{[n]_{q_{n}}}=0 .
$$

So we have

$$
s t-\lim _{n}\left\|K_{n, q_{n}}^{(\alpha, \beta)}\left(e_{1} ; \cdot\right)-e_{1}\right\|_{C[0,1]}=0 .
$$

Finally, in view of (2.7), one can write

$$
\begin{aligned}
& \left\|K_{n, q_{n}}^{(\alpha, \beta)}\left(e_{2} ; \cdot\right)-e_{2}\right\|_{C[0,1]} \\
& \leq\left|\frac{[n+p]_{q_{n}}[n+p-1]_{q_{n}}}{[n+1+\beta]_{q_{n}}^{2}} q_{n}^{2 \alpha+3}-1\right| \\
& \quad+\frac{[n+p]_{q_{n}}}{[n+1+\beta]_{q_{n}}^{2}}\left(\frac{2}{[2]_{q_{n}}} q_{n}^{\alpha+1}+q_{n}^{2+\alpha}\left(2[\alpha]_{q_{n}}+q_{n}^{\alpha}\right)\right) \\
& \quad+\frac{1}{[n+1+\beta]_{q_{n}}^{2}}\left(\frac{1}{[3]_{q_{n}}}+\frac{2 q_{n}[\alpha]_{q_{n}}}{[2]_{q_{n}}}+q_{n}^{2}[\alpha]_{q_{n}}^{2}\right) .
\end{aligned}
$$

Using (3.2),

$$
\begin{aligned}
& \frac{2}{[2]_{q_{n}}} q_{n}^{\alpha+1}+q_{n}^{2+\alpha}\left(2[\alpha]_{q_{n}}+q_{n}^{\alpha}\right) \leq 3+2 \alpha, \\
& \frac{1}{[3]_{q_{n}}}+\frac{2 q_{n}[\alpha]_{q}}{[2]_{q}}+q^{2}[\alpha]_{q}^{2} \leq(1+\alpha)^{2},
\end{aligned}
$$

and

$$
q_{n}[n+p-1]_{q_{n}}=[n+p]_{q_{n}}-1,
$$

we can write

$$
\begin{aligned}
& \left\|K_{n, q_{n}}^{(\alpha, \beta)}\left(e_{2} ; \cdot\right)-e_{2}\right\|_{C[0,1]} \\
& \quad \leq \frac{[n+p]_{q_{n}} q_{n}^{2 \alpha+2}}{[n+1+\beta]_{q_{n}}^{2}}+\left|\frac{q_{n}^{2 \alpha+2}[n+p]_{q_{n}}^{2}}{[n+1+\beta]_{q_{n}}^{2}}-1\right|+\frac{(3+2 \alpha)[n+p]_{q_{n}}}{[n+1+\beta]_{q_{n}}^{2}}+\frac{(1+\alpha)^{2}}{[n+1+\beta]_{q_{n}}^{2}}
\end{aligned}
$$




$$
\begin{aligned}
& \leq\left|\frac{q_{n}^{2 \alpha+2}[n+p]_{q_{n}}^{2}}{[n+1+\beta]_{q_{n}}^{2}}-1\right|+\frac{(4+2 \alpha)}{[n]_{q_{n}}}\left(1+\frac{[p]_{q_{n}}}{[n]_{q_{n}}}\right)+\frac{(1+\alpha)^{2}}{[n]_{q_{n}}^{2}} \\
& =: \theta_{n}+\gamma_{n}+\eta_{n} .
\end{aligned}
$$

Then, from (4.1), we have

$$
s t-\lim _{n} \theta_{n}=s t-\lim _{n} \gamma_{n}=s t-\lim _{n} \eta_{n}=0 .
$$

Here, for given $\varepsilon>0$, let us define the following sets:

$$
\begin{aligned}
& T=\left\{k:\left\|K_{n, q_{k}}^{(\alpha, \beta)}\left(e_{2} ; \cdot\right)-e_{2}\right\|_{C[0,1]} \geq \varepsilon\right\}, \\
& T_{1}=\left\{k: \theta_{k} \geq \frac{\varepsilon}{3}\right\}, \quad T_{2}=\left\{k: \gamma_{k} \geq \frac{\varepsilon}{3}\right\}, \quad T_{3}=\left\{k: \eta_{k} \geq \frac{\varepsilon}{3}\right\} .
\end{aligned}
$$

It is clear that $T \subseteq T_{1} \cup T_{2} \cup T_{3}$. So we get

$$
\begin{aligned}
\delta\{k & \left.\leq n:\left\|K_{n, q_{k}}^{(\alpha, \beta)}\left(e_{2} ; \cdot\right)-e_{2}\right\|_{C[0,1]} \geq \varepsilon\right\} \\
& \leq \delta\left\{k \leq n: \theta_{k} \geq \frac{\varepsilon}{3}\right\}+\delta\left\{k \leq n: \gamma_{k} \geq \frac{\varepsilon}{3}\right\}+\delta\left\{k \leq n: \eta_{k} \geq \frac{\varepsilon}{3}\right\} .
\end{aligned}
$$

By condition (4.5), we have

$$
\delta\left\{k \leq n:\left\|K_{n, q_{k}}^{(\alpha, \beta)}\left(e_{2} ; \cdot\right)-e_{2}\right\|_{C[0,1]} \geq \varepsilon\right\}=0,
$$

which implies that

$$
s t-\lim _{n}\left\|K_{n, q_{n}}^{(\alpha, \beta)}\left(e_{2} ; \cdot\right)-e_{2}\right\|_{C[0,1]}=0 .
$$

In view of (4.2), (4.4) and (4.6), the proof is complete.

\section{Rates of convergence}

Let $f \in C[0, p+1]$ for any $t \in[0, p+1]$ and $x \in[0,1]$. Then we have $|f(t)-f(x)| \leq \omega(f,|t-x|)$, so for any $\delta>0$, we get

$$
\omega(f,|t-x|) \leq \begin{cases}\omega(f, \delta), & |t-x|<\delta \\ \omega\left(f, \frac{(t-x)^{2}}{\delta}\right), & |t-x| \geq \delta .\end{cases}
$$

Owing to $\omega(f, \lambda \delta) \leq(1+\lambda) \omega(f, \delta)$ for $\lambda>0$, it is obvious that we have

$$
|f(t)-f(x)| \leq\left(1+\delta^{-2}(t-x)^{2}\right) \omega(f, \delta)
$$

for any $t \in[0, p+1], x \in[0,1]$ and $\delta>0$.

Now, we give the convergence rate of $K_{n, q}^{(\alpha, \beta)}(f ; x)$ to the function $f \in C[0, p+1]$ in terms of the modulus of continuity. 
Theorem 5.1 Let $q=q_{n}, 0<q_{n}<1$, be a sequence satisfying (4.1), then for any function $f \in C[0, p+1], x \in[0,1]$, we have

$$
\left|K_{n, q_{n}}^{(\alpha, \beta)}(f ; x)-f(x)\right| \leq 2 \omega\left(f, \sqrt{\delta_{n, q_{n}}^{p}(x)}\right),
$$

where $\delta_{n, q_{n}}^{p}(x)$ is given by $(2.9)$.

Proof Using the linearity and positivity of the operator $K_{n, q}^{(\alpha, \beta)}(f ; x)$ and inequality (5.1), for any $f \in C[0, p+1]$ and $x \in[0,1]$, we get

$$
\begin{aligned}
\left|K_{n, q}^{(\alpha, \beta)}(f, x)-f(x)\right| & \leq K_{n, q}^{(\alpha, \beta)}(|f(t)-f(x)| ; x) \\
& \leq\left(1+\delta^{-2} K_{n, q}^{(\alpha, \beta)}\left((t-x)^{2} ; x\right)\right) \omega(f, \delta) .
\end{aligned}
$$

Take $q=q_{n}, 0<q_{n}<1$, be a sequence satisfying condition (4.1) and choose $\delta=\sqrt{\delta_{n, q_{n}}^{p}(x)}$ in (5.2), the desired result follows immediately.

Finally, we give the rate of convergence of $K_{n, q}^{(\alpha, \beta)}(f ; x)$ with the help of functions of the Lipschitz class. We recall a function $f \in \operatorname{Lip}_{M}(\lambda)$ on $[0, p+1]$ if the inequality

$$
|f(t)-f(x)| \leq M|t-x|^{\lambda}, \quad t, x \in[0, p+1]
$$

holds.

Theorem 5.2 Let $f \in \operatorname{Lip}_{M}(\lambda)$ on $[0, p+1], 0<\lambda \leq 1$. Let $q=q_{n}, 0<q_{n}<1$ be a sequence satisfying the conditions given in (4.1). If we take $\delta_{n, q_{n}}^{p}(x)$ as in (2.9), then we have

$$
\left|K_{n, q_{n}}^{(\alpha, \beta)}(f ; x)-f(x)\right| \leq M\left(\delta_{n, q_{n}}^{p}(x)\right)^{\lambda / 2}, \quad x \in[0,1] .
$$

Proof Let $f \in \operatorname{Lip}_{M}(\lambda)$ on $[0, p+1], 0<\lambda \leq 1$. Since $K_{n, q_{n}}^{(\alpha, \beta)}(f ; x)$ is linear and positive, by using (5.3), we have

$$
\left|K_{n, q_{n}}^{(\alpha, \beta)}(f ; x)-f(x)\right| \leq K_{n, q_{n}}^{(\alpha, \beta)}(|f(t)-f(x)| ; x) \leq K_{n, q_{n}}^{(\alpha, \beta)}\left(|t-x|^{\lambda} ; x\right) .
$$

If we take $p^{\prime}=\frac{2}{\lambda}, q^{\prime}=\frac{2}{2-\lambda}$ and apply the Hölder inequality, then we obtain

$$
\left|K_{n, q_{n}}^{(\alpha, \beta)}(f ; x)-f(x)\right| \leq M\left(K_{n, q_{n}}^{(\alpha, \beta)}\left((t-x)^{2} ; x\right)\right)^{\lambda / 2} \leq M\left(\delta_{n, q_{n}}^{p}(x)\right)^{\lambda / 2} .
$$

\section{Acknowledgements}

This research is supported by the Fundamental Research Funds for the Central Universities (Nos. N1 10323010, N130323015), Science and Technology Research Founds for Colleges and Universities in Hebei Province (No. Z2014040), and the Research Fund for Northeastern University at Qinhuangdao (No. XNB201429). 


\section{References}

1. Lupaș, A: A q-analogue of the Bernstein operator. In: Seminar on Numerical and Statistical Calculus, vol. 9, pp. 85-92. University of Cluj-Napoca, Cluj-Napoca (1987)

2. Phillips, GM: Bernstein polynomials based on the $q$-integers. Ann. Numer. Math. 4, 511-518 (1997)

3. Gupta, V: Some approximation properties of q-Durrmeyer operators. Appl. Math. Comput. 197(1), 172-178 (2008)

4. Maheshwari, P, Sharma, D: Approximation by $q$ Baskakov-Beta-Stancu operators. Rend. Circ. Mat. Palermo 61(2), 297-305 (2012)

5. Aral, A, Gupta, V, Agarwal, RP: Applications of q-Calculus in Operator Theory. Springer, New York (2013)

6. Agrawal, PN, Sathish Kumar, A, Sinha, TAK: Stancu type generalization of modified Schurer operators based on q-integers. Appl. Math. Comput. 226, 765-776 (2014)

7. Gadjiev, AD, Orhan, C: Some approximation theorems via statistical convergence. Rocky Mt. J. Math. 32, 129-138 (2002)

8. Ersan, S, Doğru, O: Statistical approximation properties of q-Bleimann, Butzer and Hahn operators. Math. Comput. Model. 49, 1595-1606 (2009)

9. Dalmanoǧlu, O, Doǧru, O: On statistical approximation properties of Kantorovich type $q$-Bernstein operators. Math Comput. Model. 52(5-6), 760-771 (2010)

10. Aktuğlu, H, Özarslan, MA, Duman, O: Matrix summability methods on the approximation of multivariate $q-M K Z$ operators. Bull. Malays. Math. Soc. 34(3), 465-474 (2011)

11. Örkcü, M, Doğru, O: Weighted statistical approximation by Kantorovich type q-Szász-Mirakjan operators. Appl. Math. Comput. 217(20), 7913-7919 (2011)

12. Ren, $M-Y$, Zeng, X-M: Some statistical approximation properties of Kantorovich-type $q$-Bernstein-Stancu operators J. Inequal. Appl. 2014, Article ID 10 (2014)

13. Vedi, T: Some Schurer type $q$-Bernstein operators. Master's thesis, Eastern Mediterranean University (2011)

14. Muraru, CV: Note on the q-Stancu-Schurer operator. Miskolc Math. Notes 14(1), 191-200 (2013)

15. DeVore, RA, Lorentz, GG: Constructive Approximation. Springer, Berlin (1993)

16. Fast, H: Sur la convergence statistique. Colloq. Math. 2(3-4), 241-244 (1951)

17. Niven, I, Zuckerman, HS, Montgomery, HL: An Introduction to the Theory Numbers, 5th edn. Wiley, New York (1991)

18. Doğru, O: On statistical approximation properties of Stancu type bivariate generalization of $q$-Balás-Szabados operators. In: Seminar on Numerical Analysis and Approximation Theory, pp. 179-194. University Babeş-Bolya, Cluj-Napoca (2006)

10.1186/1029-242X-2014-465

Cite this article as: Lin: Statistical approximation of modified Schurer-type $q$-Bernstein Kantorovich operators. Journal of Inequalities and Applications 2014, 2014:465

\section{Submit your manuscript to a SpringerOpen ${ }^{\ominus}$ journal and benefit from:}

- Convenient online submission

- Rigorous peer review

Immediate publication on acceptance

Open access: articles freely available online

- High visibility within the field

- Retaining the copyright to your article 